\title{
Subject Index to Volume 17
}

Absorption, jejunal 381

Acetylcholine 331

Achalasia 532

Acinar cells 899

Acrodermatitis enteropathica 239, 354

ACTH insensitivity 532

ACTH tests 861

Acute renal failure 985

Acyl-CoA dehydrogenation deficiencies 828

Adenosine 331

Adenosine deaminase 935

Adenylate cyclase 101, 553

Adrenal gland 131, 362, 861

$\alpha$-Adrenergic blockage 464

Adrenergic receptor 523

$\beta$-Adrenergic receptor 213,553

$\alpha$-Adrenoceptors 177

Aerosolization, jet 742

Aerosolization, ultrasonic 742

Agar 810

Airway 508

Alacrima 532

Albumin 141, 850

Aldosterone 195

Allergy, milk 406

Alzheimer II 970

Amino acid 704, 710, 733

Aminophylline 188,331

Ammonia 729

Amniotic fluid 541, 976

Amrinone 720

Amylase 806, 998

Amylase, mammary 15

Anemia 70, 168, 503

Angiotensin 195, 210, 331

Angiotensin I 762

Angiotensin-converting enzyme

Angiotensin, kidney 210

Anophthalmia 313

Antibodies, adrenal 861

Antibody, jejunum 406

Antibody, passive 707, 724

Antigen, enteric 449

Apnea 35, 188, 213, 550, 592

Apoprotein 83, 439

Arginase 941

Arginine vasopressin 583

Arginine vasotocin 583

Arylsulfatase A deficiency 701

(Sept)

Aspartate aminotransferase 884

Aspiration 976

Asthma 508

Astrocytes 970

Ataxia 25

Atropine 331

Autoimmune adrenalitis 861

Autonomic nervous system 162, 491
Autoregulation 246

Behavior 872

Behavior, sleep 802

Beta cell 758

Betamethasone 111

Bicarbonate 486

Bile 716

Biliary atresia 296

Bilirubin 120, 192, 208, 888

Bilirubinometry 888

Biotin 61

Blood, cord 57, 474

Blood, viscosity 452

Blood flow 908, 945

Blood flow, cerebral 70, 77, 246, 908

Blood flow, organ 919

Blood flow, systemic 254

Bone 204, 259

Bradycardia 945

Brain 8, 344, 349, 514, 606

Brain, blood flow 70, 77, 246, 908

Breath test 629

Bromocriptine 665

Bronchiolitis 753

Bronze baby syndrome 327

Caffeine 592

Calcium 784, 856, 912

Carbohydrate $15,124,157,629$, 733, 931

Carbonic anhydrase 390

Cardiac output 903, 919

Cardiomyopathy 319

Cardiopulmonology 680

Carnitine 579, 877

Carnitine deficiency 319

Casein 912, 1008

Catecholamine 456

Cerebroside sulfate 701 (Sept)

Charcoal 208

Chemotaxigenesis 496

Chemotaxis 57, 183, 292, 993

Chloride 267

Chlorothiazide 117

Cholera toxin 553

Cholestasis 327

Cholesterol 124, 689, 770, 788

Cholesterol ester storage disease 770

Chromosome 398

Chromosome, abnormality 1

Chromosome, translocation 398

Chronic granulomatous disease 916

Cimetidine 820

Circulation, kidney 620

Circulation, scalp 982

Clearance methods 1013

Clindamycin 916
Coagulation 482

Coffin-Lowry syndrome 926

Colonization 472

Colostrum 825,868

Coma 959

Complement 296, 685, 952

Congenital adrenal hyperplasia 131

Congenital defects 263

Consumption 729

Copper 784, 912

Cor pulmonale 720

Corticosteroid 766

Cyclic AMP 959

Cyclooxygenase 107

Cystic fibrosis 30, 65, 181, 267, 486, 523, 701, 747, 850, 899, 931,952

Cytochrome P-450 828

Cytokine 868

Cytotoxicity 868

Dehydration 919

Dehydroepiandrosterone sulfate 131

Depressor effect 924

Development, embryonic 313

Dexamethasone 274

Diabetes mellitus 101, 200, 587

Diagnosis, prenatal 541

Diarrhea 381

Dibutyryl cyclic-AMP 331

Dicarboxylic acid 828, 877

Dicarboxylic aciduria 171

Diet 319

Disaccharide 381

Diuresis 1013

L-Dopa 924

Dopa decarboxylase 924

Dopamine 464

Down's syndrome 292

Ductus arteriosis 331

Energy, storage 891

Enkephalin 162

Enteropathies 406

Enterotoxin 1008

Epinephrine, synthesis 362

Erythrocyte 250, 376, 452, 567, $579,672,935$

Escherichia coli 349, 1017

Estradiol 689

Estrogen 1

Ethanol 529, 704

Excretion 737

Exercise 267

Familial erythrophagocytic lymphohistiocytosis 413

Fanconi syndrome 157, 710

Fasting 285, 319
Fat $220,634,716,891$

Fat, brown 362

Fatty acid 61, 171, 224, 877

Fetal alcohol syndrome 529, 704

Fetal lung 596

$\alpha$-Fetoprotein 47

Fibroblast 97, 239, 541

Fibronectic 482

Fluphenazine 665

Folic acid 61

Free fatty acids 634

Fumarylacetoacetate fumarylhydrolase 541

Furosemide 141

Gastroenterology, developmental 19

Gastrointestinal barrier 856

Galactose 157, 609, 931

Ganglioside 413

Gastrointestinal tract 19

Gaucher's disease 344

Genetic damage 192, 234

Glomerulus 762

Glucagon 51, 101

Glucocorticoid 111, 672

Glucose 51, 285, 319, 634, 729, 758,998

Glucose-6-phosphatase translocase 545

Glucosylceramide 344

Glutathione 562

Glycogen 587, 766

Glycogenolysis 639

Glycogen phosphorylase 587

Glycogen storage disease 157, 545

Glycogen synthase 587

Glycolysis 639

Glyconeogenesis 639

Glycoprotein 30,65

Glycosylation 200

Gonadotropin 676

Granulocyte 57

Granulocytic stem cell 278

Growth 676

Growth hormone 592, 676

Growth retardation 602

Guanine nucleotides 553

Heart 263, 319, 567, 680

Heart rate $267,491,651,872,903$

Heat, body 135

Hemoglobin 200

Hemoglobin, corpuscular 168

Hemoglobin, unstable 503

Hemophilus influenzae 472

Hemorrhage 70

Hepatic catalase 714

Hepatic uricase activity 714

Hepatitis 296, 814

Hepatocyte 609 
Hepatosplenomegaly 307

Histamine 820

Hydrocarbon pneumonitis 657

Hydrocortisone 131

21-Hydroxylase deficiency 131

4-Hydroxyphenylpyruvate deoxygenase deficiency 25

Hyperactivity 802

Hyperammonemia 970

Hyperbilirubinemia 461, 810

Hypercalciuria 117

Hypercapnia 567

Hyperglycemia 51

Hyper-IgE syndrome 820

Hyperketonemia

Hyperlipoproteinemia 835

Hypernatremia 919

Hyperoxia 368

Hypertyrosinemia 92

Hypoalbuminemia 850

Hypocarbia 70

Hypocortisolism 861

Hypoglycemia 171, 319,877

Hypoketonemia 319

Hyponatremia 606

Hypotension 77

Hypothermia 945

Hypothyroidism 8, 111, 468

Hypoxemia 195, 254

Hypoxia 567

IgE 820

IgG 931

Ileum 1008

Imidazole 331

Immunity 338,935

Immunity, cellular 753,820

Immunity, humoral 753,820

Immunization 449

Immunization, oral 707,724

Incubator 135

Indomethacine 331

Infection, bacterial 685

Influenza 338

Injury, tissue 482

Insulin $111,672,758$

Interferon 573,868

Intestinal transport 733

Intestine $65,148,354,537,625$

Intralipid 478

Intrauterine growth retardation 704

Iodine 8

Iodocompounds 8

Ion content, brain 606

Iron 168,912

Isoenzymes, mitochondrial 884

Jejunum 406

Jejunum, absorption 381

Kallikrein 737

Ketogenesis 224, 877

Ketone 51

Kidney 42, 141, 177, 390, 514, $620,737,762,766,985,1013$

Krebs cycle 639

Lactate 729
Lactose 629,825

Larynx 550

Laser therapy 120

Leukemia, acute 398

Leukocyte 97, 916

Leukodystrophy 701

Lipase 716, 770, 806

Lipid $61,83,124,344,742,835$

Lipoprotein $689,788,835$

Liquid ventilation 680

Liver 101, 111, 224, 285, 413, 514, $541,562,609,714,1017$

Liver, metabolism 639

Liver disease 296, 319

Lung $30,230,274,303,338,362$, $368,439,508,553,657,680$, $720,747,959,976,1002$

Lymphocyte 474, 541, 573, 799, 820,935

Lymphohistiocytosis 413

Lymphokine 573, 799

Lysozyme 241

Macrophages, milk 241

Magnesium 263, 784, 912

Maleic acid syndrome 710

Malformations 313

Malformations, congenital 83

Malnutrition 806

Malnutrition, fetal 704

Mannitol 583

Meconium 976

Medium-chain acyl-CoA dehydrogenase 877

$\alpha$-Melanocyte 903

Metabolism, brain 285

Metabolism, liver 285

Metachromatic leukodystrophy 701 (Sept)

Methoxamine 331

4-Methylumbelliferyl p-quanidinobenzoate 850

Methysergide 331

Microphthalmia 313

Microvillus 856

Milk, cow 406

Milk, human 61, 241, 468, 716, $825,835,891,912$

Minerals 784

Mitochondrion, isoenzymes 884

Mitogen 474

Monocarboxylic acids 828

Monocyte 183, 292

Monooxygenase 828

Mucin 65, 899

Multiple sulfatase deficiency 97

Multivitamin 192

Muscle 602

Myocardium, function 567

Necrosis, brain 349

Neonate 976

Nephrotic syndrome 733

Nerve, vagus 491

Nerve, cardiac 491

Neural system 8

Neural tube defects 47

Neurons 970

Neutropenia 278, 795
Neutrophil 183, 281, 358, 662, $795,814,820,993$

Niacin 61

Nitrogen 891

Norepinephrine 331

$5^{\prime}$ Nucleotidase 474

Nutrition 579, 602

Nutrition, newborn 478

Nutrition, parenteral 478

Occipito-frontal circumference 949

Opsonization 952

Osteoblasts 204

Osteogenesis imperfecta tarda 204

Ouabain 220

$\omega$-Oxidation 828

Oxidation, fatty acid 877

Oxygen 195, 368, 567, 729

Oxygen, consumption 254, 514

Oxygen, toxicity 181

Oxygenation, arterial 246

Pancreas 486, 758

Pancreas, enzymes 806

Pepsin 15

Peptides, chemotactic 993

Petechiae, lung 230

Phagocyte 814

Pharmaceutical 617

Phentolamine 331

Phenylethanolamine $N$-methyl transferase 362

Phospholipids 742

Phototherapy 120,192, 208, 234 $461,810,888$

Picolinic acid 239

Pituitary gland 592, 676

Placenta 449

Placenta, growth 587

Placenta, human 729

Pneumonia 753

Pneumonitis, hydrocarbon 657

Polymorphonuclear leukocytes 496

Porphyrin 327

Precolostrum 825

Prednisone 672

Prolactin 665

L-Proline 42

Propanol 720

Propranolol 331

Prostacyclin 537

Prostaglandin 107, 331

Prostaglandin $E_{2} 241,537,669$

Protein 124, 24

Protein, kinase 959

Protein, milk 707, 724

Protein, phosphorylation 959

Proteoglycans 926

Pseudomonas aeruginosa 747, 952

Pulmonary vasculature 720

Pulsatility index 908

Receptors, $\beta$-adrenergic 274

Receptors, pituitary 270

Renin 210, 762

Renin, high molecular weight 376

Reserpine 486, 523
Respiration 152, 188, 213, 508, 550

Respiration, sleep 35

Respiratory distress syndrome 35 , $162,448,742$

Respiratory failure 567

Respiratory syncytial virus 753

Retardation, psychomotor 307

Reticuloendothelial system 482

Reye's syndrome 877,884

Rhinitis 472

Riboflavin 234

Rifampin 916

Rotavirus 868

Scalp, blood flow 982

Screening 296

Sepsis 278, 795

Septic shock 482

Serotonin 331

Severe combined immunodeficiency 935

Shock 919

Sialic acid 307, 413, 931

Skull 949

Sleep states 35,152

Social interaction, parent-infant 872

Sodium 267, 737, 1013

Speech, infant 625

Staphylococcus 657, 916

Starvation 634

Starvation, maternal 639

Stimulating hormone 903

Stimuli, auditory 802

Stomach 669, 998

Streptococcus, group B 107, 278, $358,496,685,795,1002$

Streptozotocin 587

Stress 358

Stress, heat 267

Sublingual gland 523

Substance P 737

Sudden infant death syndrome 230,784

Sulphamethoxazole 820

Sulfamethozole 916

Superoxide 281, 662

Superoxide dismutase 181

Surfactant 439, 448

Sweat gland 701 (Aug)

Synaptogenesis 8

Tachycardia 651

$\Upsilon$ cell 573

Temperature, ambient 254

Testosterone 689

Theophylline 532, 592

Thermogenesis 220

Thyroid gland 592

Thyroid hormones 468

Thyroid releasing hormone 270

Thyroid stimulating hormone 270

Thyrotropin 592

Thyrotropin-releasing hormone 592

Thyroxine $562,592,596$

Tonsure effect 982

Toxemia 1002 
Trachea 338

Transcutaneous 888

Trauma 482

Triglyceride 319

3, 5, 3' Triiodothyronine $\left(\mathrm{T}_{3}\right) 596$

$3,5^{\prime}, 3^{\prime}$ Triiodothyronine $\left(\mathrm{rT}_{3}\right)$ 596

Trimethoprim 820,916

Turner's syndrome 1
Tyrosine 541

Tyrosinemia 25, 92

Tyrosinemia, hereditary 541

Ulcer 669

Ultrasound, Doppler 908

Uranyl nitrate 985

Urogastrone 825
Vasopressin, arginine 583

Ventilation, liquid 303

Vibrio cholerae 1008

Virus 472

Vitamin $\mathrm{D}_{2}$ 97, 117

Vitamins, B 61

Volume, skull 949

Water, brain 606
Water, tissue 665

Water, transfer 583

Whey proteins 912

Wolman's disease 770

Zellweger syndrome 514

Zinc 148, 239, 354, 529, 562, 784

912 